\section{Soplo normal, inocente o inorgánico}

\section{Normal, innocent or inorganic murmur}

\author{
Luis Alfonso Fonseca-Sánchez ${ }^{1}$ \\ Alfredo Bobadilla-Aguirre ${ }^{2}$ \\ Jorge Espino-Vela ${ }^{3}$ \\ ${ }^{1}$ Cardiólogo Pediatra. \\ 2 Jefe del departamento de Cardiología Pediátrica. \\ ${ }^{3}$ Editor emérito de Acta Pediátrica de México. \\ Instituto Nacional de Pediatría, México.
}

\section{DEFINICIÓN}

Un soplo cardiaco es un fenómeno acústico ocasionado por el cambio del flujo sanguíneo laminar a flujo turbulento; ${ }^{1,2}$ ocurre durante el paso de sangre a través de estructuras cardiacas o vasculares como defectos septales, válvulas o en la bifurcación de los vasos. Un soplo normal se produce en ausencia de cardiopatía y de manifestaciones clínicas. Se debe al incremento de velocidad del flujo sanguíneo o al cambio en el diámetro de las estructuras cardiacas. Aunque se prefiere usar el término normal también es conocido como soplo inocente, inorgánico o fisiológico. ${ }^{3}$

\section{EPIDEMIOLOGÍA}

Hasta $80 \%$ de los recién nacidos y cerca de $60 \%$ de los niños escolares pueden presentar soplos. ${ }^{1,4}$ De hecho, se considera que todos los niños presentarán algún soplo durante su desarrollo normal. $^{3}$

\section{CARACTERÍSTICAS GENERALES DE LOS SOPLOS NOR-} MALES

Los soplos normales pueden identificarse fácilmente si se conocen sus características en común como la ausencia de síntomas, momento del ciclo cardiaco en el que se presentan, intensidad y comportamiento:

1. Análisis clínico: el interrogatorio es muy importante para el diagnóstico de un soplo normal. No deben existir síntomas de cardiopatía ni signos clínicos de insuficiencia cardiaca o cianosis.
Recibido: 7 de febrero del 2014

Aceptado: 10 de diciembre del 2014

Correspondencia: Dr. Luis Alfonso Fonseca Sánchez

Emiliano Zapata 60

Santa Anita, Iztacalco

México, D.F.

Teléfono: 55304132

cyclops_luis@hotmail.com

Este artículo debe citarse como

Fonseca-Sánchez LA, Bobadilla-Aguirre A, EspinoVela J. Soplo normal, inocente o inorgánico. Acta Pediatr Mex 2015;36: 50-54. 
Fonseca-Sánchez LA et al. Soplo normal, inocente o inorgánico

2. Momento: los soplos normales son sistólicos a excepción del zumbido venoso, que es continuo. Cualquier soplo diastólico debe considerarse patológico y se recomienda realizar estudios de apoyo como radiografía de tórax, electrocardiograma y ecocardiograma. ${ }^{4}$

3. Intensidad: en 1933 Levine $^{5}$ clasificó los soplos en 6 grados de acuerdo con su intensidad (Cuadro 1). Los soplos normales son de intensidad igual o menor al grado II.

4. Ausencia de fenómenos agregados: un soplo normal no ocupa la totalidad de la sístole, por lo que el primer y segundo ruidos están conservados. El desdoblamiento fisiológico y no constante del segundo ruido cardiaco se conserva y no existen otros fenómenos agregados como chasquidos.

5. Comportamiento ante algunas maniobras: los cambios de posición como poner al paciente de pie, la maniobra de Valsalva o la actividad física pueden disminuir la intensidad del soplo o incluso lo pueden hacer desaparecer.

Cuadro 1. Clasificación de los soplos de acuerdo con su intensidad

\begin{tabular}{|c|c|}
\hline Grado I & $\begin{array}{l}\text { Soplo muy tenue, apenas audible para oídos } \\
\text { expertos; requiere escuchar varios latidos } \\
\text { para identificarlo }\end{array}$ \\
\hline Grado II & $\begin{array}{l}\text { Soplo más intenso que el anterior, fácilmente } \\
\text { identificable }\end{array}$ \\
\hline Grado III & Soplo intenso sin frémito \\
\hline Grado IV & Soplo intenso acompañado de frémito \\
\hline Grado V & $\begin{array}{l}\text { Soplo intenso con frémito más intenso que el } \\
\text { anterior, audible a través de estructuras sólidas } \\
\text { y audible al colocar el borde del estetoscopio }\end{array}$ \\
\hline Grado VI & $\begin{array}{l}\text { Soplo intenso, con frémito acentuado y au- } \\
\text { dible aún sin colocar el estetoscopio sobre } \\
\text { el tórax }\end{array}$ \\
\hline
\end{tabular}

Estudios adicionales. Existe controversia sobre la necesidad de estudios adicionales ante un soplo normal. Muchos autores consideran que si se trata de un soplo de características normales en un niño asintomático, con peso y talla normales, no se requieren más estudios ${ }^{2}$ pues no generan diferencia significativa en el diagnóstico. ${ }^{6,7}$

El examen físico realizado por un pediatra tiene sensibilidad de $86 \%$ y especificidad de $61 \%$. El examen físico más una radiografía de tórax y un electrocardiograma tienen sensibilidad de $88 \%$ y especificidad de $83 \% .{ }^{8}$ Por ello otros autores recomiendan que el estudio del soplo normal se complemente con dichos estudios.

\section{Tipos de soplo normal más comunes}

En un corazón normal el paso de la sangre a través de válvulas y vasos sanguíneos produce un flujo laminar y no genera soplos (Figura 1A). Sin embargo, existen zonas denominadas "conexiones desproporcionadas de tamaño"2 donde la disminución súbita del diámetro en las estructuras vasculares (por ejemplo la conexión de ventrículo izquierdo a aorta) produce aceleración del flujo y vibraciones que causan flujo turbulento y contribuyen a la formación de un soplo.

Los cinco sitios donde ocurren cambios acentuados en el diámetro de las estructuras cardiacas determinan los tipos más comunes de soplo normal en la edad pediátrica:

\section{Soplo de Still o vibratorio}

Se produce en la conexión del ventrículo izquierdo con la aorta. ${ }^{2}$ Es un soplo de tono musical ${ }^{1-3}$ común en pacientes entre los tres años y la adolescencia. ${ }^{3}$ Se ausculta en el mesocardio y en el ápex (Figura 2), se inicia poco después del primer ruido y ocupa la primera mitad de la sístole, es de 
baja intensidad (grado I o II) y no borra los ruidos normales (Figura 1B); se intensifica en decúbito dorsal y desaparece o disminuye con los cambios de posición. El diagnóstico diferencial es con el soplo de la comunicación ventricular que se irradia a la derecha del esternón y oscurece el primer ruido cardiaco, puede durar parte o toda la sístole ${ }^{1}$ (Figura 1C).

\section{Soplo pulmonar}

Se debe al paso de sangre del ventrículo derecho a la arteria pulmonar. ${ }^{2}$ Es el soplo más frecuente en la edad pediátrica, ${ }^{1}$ particularmente común en adolescentes y en pacientes con tórax excavado. ${ }^{2}$ Se ausculta en el foco pulmonar (Figura 2) durante la primera mitad de la sístole (Figura 2D) y puede irradiar de forma vertical. Se incrementa con el ejercicio, la fiebre y otros estados hipercinéticos. ${ }^{3}$ Disminuye o desaparece con la inspiración profunda y con la maniobra de Valsalva. ${ }^{2,3}$ El diagnóstico diferencial es con el soplo de la comunicación interauricular que se acompaña de un segundo ruido desdoblado constantemente en el foco pulmonar (Figura 1E) o con el soplo de estenosis pulmonar, que será de cualidad áspera y puede acompañar frémito palpable también en el foco pulmonar (Figura 1F).

\section{Zumbido venoso yugular}

Se origina en la unión de la vena yugular, la subclavia o la innominada a la vena cava superior. ${ }^{2}$ Es frecuente entre los 3 y los 8 años de edad. Es el único soplo normal que es continuo y se acentúa en la diástole (Figura 1G). Frecuentemente se ausculta en el trayecto de la vena yugular derecha, aunque puede ser bilateral (Figura 2); se identifica mejor con el paciente de pie; aumenta con el ejercicio, la fiebre y la anemia; desaparece en el decúbito y con la maniobra de Valsalva, lo que ayuda a diferenciarlo del soplo del conducto arterioso permeable que no desaparece con el decúbito y se atenúa en la diástole (Figura $1 \mathrm{H}$ ).

\section{Soplo sistólico carotídeo o soplo de flujos sistémicos}

Es producido en la conexión de la aorta con los vasos supraórticos. Se ausculta en el trayecto de la carótida derecha principalmente, aunque puede ser bilateral (Figura 2). Se escucha durante la mitad sístole, con inicio y fin súbito (Figura 1I), es poco audible en el foco aórtico y más intenso en el trayecto de la carótida, lo que ayuda a diferenciarlo del soplo de estenosis aórtica que es sistólico con máxima auscultación en el foco aórtico, precedido de un clic de eyección, puede irradiarse a cuello y acompañarse de frémito, además el componente aórtico del segundo ruido puede estar disminuido o ausente, por lo que el segundo ruido puede ser único. (Figura 1J).

\section{Estenosis pulmonar periférica del neonato}

Se origina en la bifurcación del tronco de la arteria pulmonar. ${ }^{2}$ Se considera propia del neonato, principalmente en prematuros. Su principal sitio de auscultación es el borde esternal izquierdo, con irradiación a las regiones infraclaviculares, axila y espalda. Desaparece durante el primer año. ${ }^{2}$ Inicia poco después del primer ruido y no ocupa la totalidad de la diástole (Figura $1 \mathrm{~K}$ ).

\section{ACTITUD ANTE UN SOPLO}

El hallazgo de un soplo durante la exploración pediátrica suele generar preocupación en los familiares. Por ello en primer lugar se debe tener la seguridad de identificar a un soplo normal. ${ }^{4}$ Se debe realizar una historia clínica completa 
Fonseca-Sánchez LA et al. Soplo normal, inocente o inorgánico

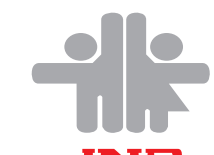

INP

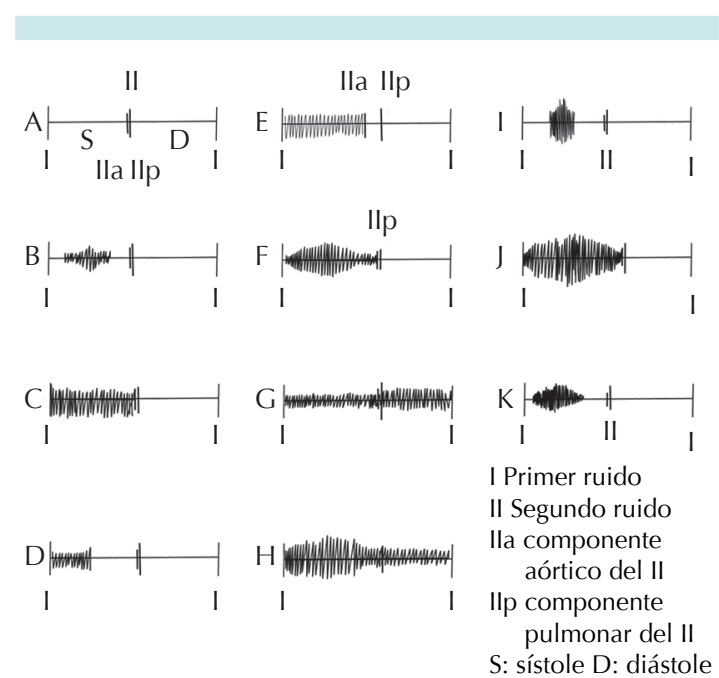

Figura 1. Características de los soplos normales durante la auscultación y sus principales diagnósticos diferenciales. A) Ruidos cardiacos normales. Audibles en todos los focos, sin fenómenos agregados. B) Soplo de Still audible en la zona comprendida entre el mesocardio y el ápex, ocupa la mitad de la sístole, es de intensidad baja y no tiene irradiaciones. C) Soplo de la comunicación ventricular. Audible en mesocardio, con irradiación en barra, ocupa toda la sístole y puede ocultar al segundo ruido. D) Soplo inocente pulmonar. Audible en el foco pulmonar, ocupa la mitad de la sístole, es de intensidad baja y sin frémito. E) Soplo de la comunicación interauricular. Audible en el foco pulmonar, ocupa toda la sístole y se acompaña de un segundo ruido desdoblado constantemente. F) Soplo de estenosis pulmonar. Audible en el foco pulmonar, ocupa toda la sístole, puede acompañarse de frémito. G) Zumbido venoso. Audible en el trayecto de la vena yugular, es el único soplo inocente continuo, es más intenso en la diástole. H) Soplo del conducto arterioso permeable. Audible en el foco pulmonar, es continuo pero más intenso en la sístole. I) Soplo sistólico carotideo o soplo de flujos sistémicos. Audible en el trayecto de las carótidas, de inicio y fin súbito a mitad de la sístole. J) Soplo de estenosis aórtica. Su sitio de máxima auscultación es el foco aórtico, ocupa toda la sístole. K) Soplo de estenosis pulmonar periférica del neonato. Audible en el borde paraesternal izquierdo, ocupa la mitad de la sístole, se escucha durante el primer año de vida.

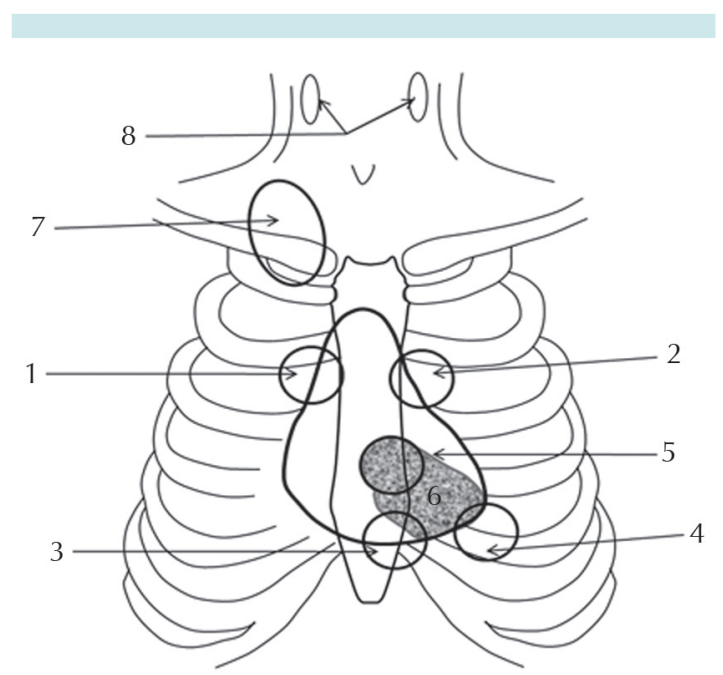

Figura 2. Principales focos y áreas de auscultación pediátrica. 1. Foco aórtico. 2. Foco pulmonar. 3. Foco tricuspídeo. 4. Foco mitral. 5. Mesocardio. 6. Área donde se ausculta el soplo de Still (sombreada). 7. Área donde se ausculta el zumbido venoso. 8. Trayecto de la carótida, área donde se ausculta el flujo sistólico carotídeo.

y un interrogatorio específico de síntomas cardiacos como diaforesis, fatiga a la alimentación, taquipnea, disnea, cianosis, síncope, etc. La exploración física debe ser minuciosa: inspección de características físicas, palpación de pulsos, choque de la punta y área cardiaca. La auscultación es fundamental para identificar un soplo normal, se deben buscar sus características generales y especificas. Una vez establecido el diagnóstico de soplo normal se debe informar a los familiares la naturaleza del soplo y su carácter benigno. Todo soplo que no tenga características de normal requiere que el paciente sea referido a un centro hospitalario para su estudio, pues la precisión del diagnóstico determinará el tratamiento y evitará posibles complicaciones. De igual forma, ante duda de la "normalidad" 
de un soplo se sugiere referir al paciente a un especialista para su confirmación.

\section{CONCLUSIÓN}

Un soplo es un hallazgo frecuente en la consulta pediátrica. Los soplos normales se presenta en pacientes asintomáticos y sin cardiopatía. La principal herramienta para diagnosticarlos es la clínica, identificar las características generales y especificas de cada uno de ellos es suficiente para realizar el diagnóstico.

\section{REFERENCIAS}

1. Guadalajara BJ. Hernández JG. Cervantes EJ. GonzálezHermosillo GJ. Colín LL. Alexanderson E. Historia clínica.
En: Guadalajara BJ. Hernández JG. Cervantes EJ. GonzálezHermosillo GJ. Colín LL. Alexanderson E. Cardiología. 5a edición. Méndez Editores. México 2003. P 25-59.

2. Sapin SO. Recognizing Normal Heart Murmurs: A Logicbased Mnemonic. Pediatrics 1997;99(4):616-619.

3. Biancaniello T. Innocent Murmurs. Circulation 2005;111:e20e22.

4. Duhagón P. Soplos cardíacos. Arch Pediatr Urug 2002;73(1):22-25.

5. Levine SA. The systolic murmur: its clinical significance. JAMA 1933;101:436-438.

6. Frank JE and Jacobe KM. Evaluation and management of heart murmurs in children. Am Fam Physician 2011;84(7):793-800.

7. Danford DA. Heart murmur in a Child. JCOM 2002;9(3):146158.

8. Flores TL, Garrido GL. Estudio de los soplos cardiacos en la edad pediátrica. Acta Pediatr Mex 2012;33(5):252 257.

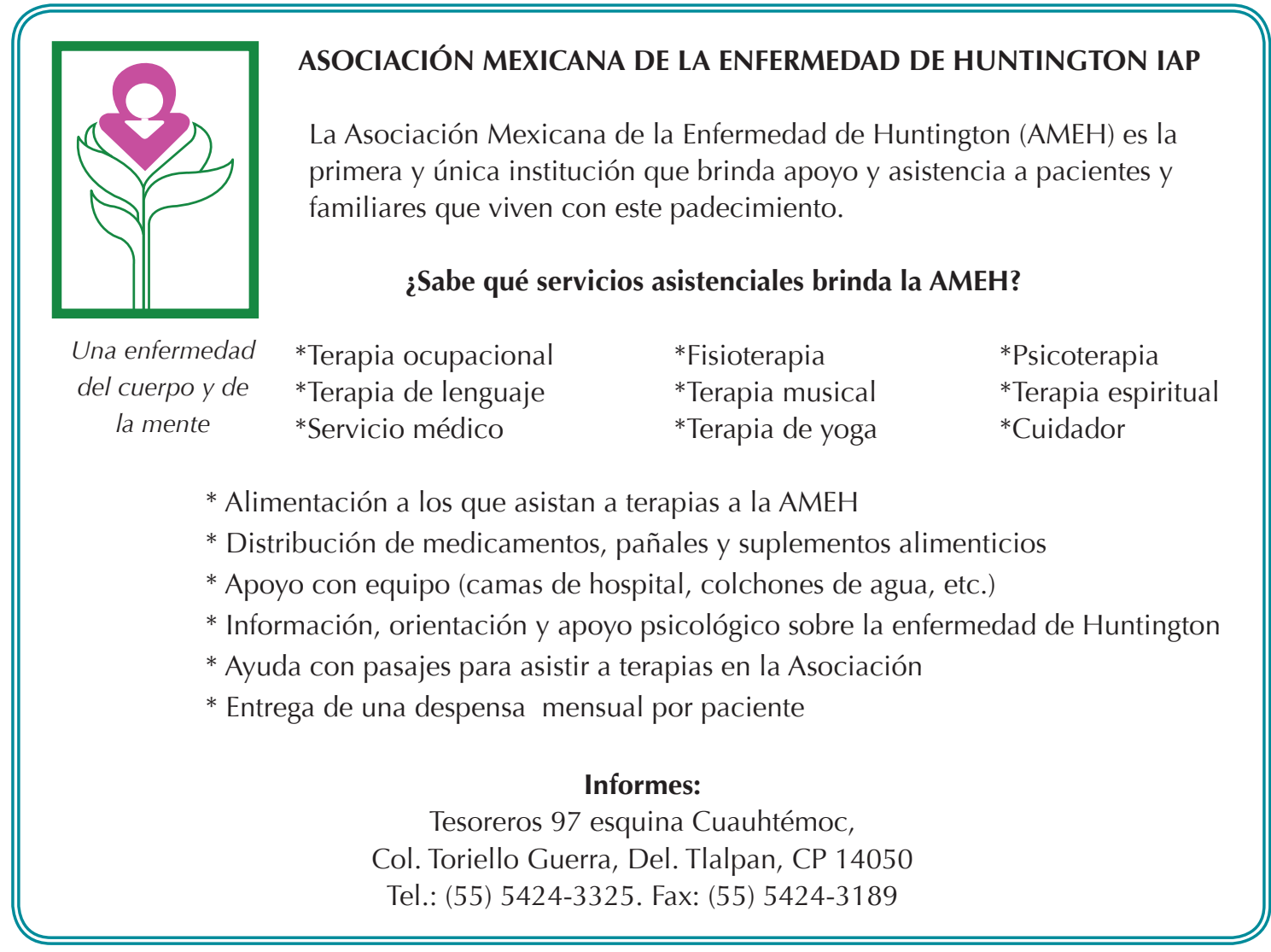

